\title{
Football Player Tracking for Video Annotation
}

\author{
P. Huang and A. Hilton
}

Centre for Vision Speech and Signal Processing, University of Surrey, Guildford, GU2 7XH, UK

Keywords: Video Analysis, Tracking, Optimisation

\section{Introduction}

This paper presents a method to combine unreliable player tracks from multiple cameras to obtain a unique track for each player. A recursive graph optimisation algorithm is introduced to evaluate the best association between player tracks.

Annotation of sports video to illustrate player movements for commentary and extract play statistics is of considerable interest for broadcast production. The BBC Piero system provides single view online tracking using match cameras (http://www.bbc.co.uk/rd/projects/virtual/piero/).

The Ascencio multiple camera system requires 32 man-hours to analyse a complete match (http://www.footballpro.com/). Football player tracking is a challenging problem due to the rapid movement and occlusion between players [3, 1, 2]. Football player tracking from multiple camera views leads to a coupled problem of data association (tracking) and reconstruction (player location). In this work we propose a recursive graph optimisation algorithm to combine individual player tracks from multiple views using the ground-plane constraint to estimate player location. This approach provides an online algorithm to obtain unique tracks for each player without manual intervention. Initial results indicate that this approach is promising.

\section{Single View Player Tracking}

Single view tracking of football players is inherently ambiguous due to both frequent player-player occlusion and rapid changes in player direction. In this work we seek to obtain short single view player tracks when players are un-occluded and combine these across multiple views to obtain continuous player tracks. Single view tracking is performed in three steps: background matting; colour clustering; and labelling. Initially colour based foreground background matting is applied to obtain a foreground mask for the players. Gaussian Mixture Model clustering is then applied to the foreground mask to segment distinct colour regions corresponding to player shirts for each team. Temporal labelling of colour regions is then performed using a nearest neighbour criteria to associate regions in successive frames. Tracks are terminated where multiple single view tracks converge due to player-player occlusion, Figure 1.

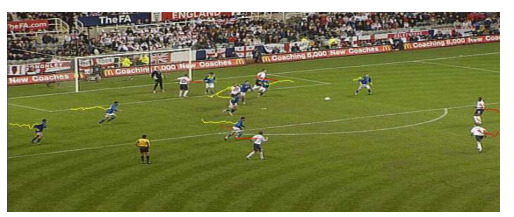

Figure 1: Single view player tracks (frame 75)

\section{Integration of Player Tracks for Multiple Views}

Due to player-player occlusion single view tracking cannot reliably obtain player tracks for the entire game. In this work we investigate the combination of player tracks from multiple calibrated camera views using a graph optimisation framework. Player location on the pitch from each view is estimated by projection of the player shirt region centroid onto a plane at $1.5 \mathrm{~m}$ height above the pitch ground-plane. Clustering of player locations between views is then performed to approximate the player location. A graph representation of the multiple view player tracks is constructed by combining short player tracks from multiple views as nodes with branches where multiple cluster tracks corresponding to different players intersect. Unique tracks for each observed player at a given time instant, $t$, can then estimated as the shortest path to players observed on the first frame $t=0$ which is solved using Dijkstras algorithm. To improve efficiency the shortest path player labelling can be fixed at an intermediate time, $t-\tau$, where all players are uniquely observed. Initial evaluation of the recursive graph optimisation algorithm for multiple view player tracking has been performed on a sequence of $200 \mathrm{PAL}$ resolution fields of professional football captured with 15 static cameras viewing half the pitch. Table 1 and Figure 2 show the percentage of correctly labelled players for different delays $\tau$ measured against ground-truth hand labelling. Currently both approaches require improvement to achieve correct labelling on all frames.

In conclusion, a recursive graph optimisation algorithm has been investigated for combining player tracks from multiple views to obtain unique labelling. The combination of multiple views resolves many of the visual ambiguities due to occlusion in single view tracking. Further work is required to achieve fully automated player tracking and utilise match cameras rather than specialist camera systems.

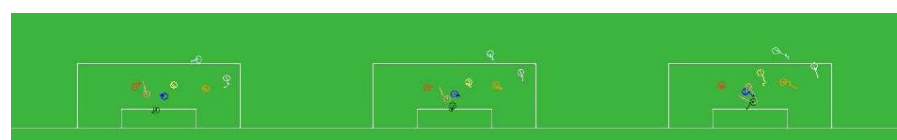

Figure 2: Multi-view player tracks overhead view (frames $60,75,90$ )

\begin{tabular}{l|c|c|c|c}
\hline & $\begin{array}{c}\text { Global } \\
\text { (off-line) }\end{array}$ & $\begin{array}{c}\text { Delay=90 } \\
\text { (on-line) }\end{array}$ & Delay = 30 & Delay = 10 \\
\hline Correct Rate & $85.43 \%$ & $74.44 \%$ & $68.31 \%$ & $34.63 \%$ \\
\hline
\end{tabular}

Table 1: Evaluation of multiple view tracking

\section{References}

[1] J. Sullivan, S. Carlsson and E. Hayman. "Tracking and Labelling of Interacting Multiple Targets”. ECCV 2006.

[2] M. Xu, J.Orwell and G.Jones. "Tracking Football Players with Multiple Cameras". ICIP 2004.

[3] A.Dearden, Y.Demiris and O.Grau. "Tracking Football Player Movement from a Single Moving Camera using Particle Filters". CVMP 2006. 